\title{
Article \\ Differential Geometry Approach to Continuous Model of Micro-Structural Defects in Finite Elasto-Plasticity
}

\author{
Sanda Cleja-Ţigoiu ${ }^{1,2}$
}

1 Faculty of Mathematics and Computer Science, University of Bucharest, 010014 Bucharest, Romania; tigoiu@fmi.unibuc.ro

2 Research Institute of the University of Bucharest, University of Bucharest, 050657 Bucharest, Romania

Citation: Cleja-Ţigoiu, S. Differential Geometry Approach to Continuous Model of Micro-Structural Defects in Finite Elasto-Plasticity. Symmetry 2021, 13, 2340. https://doi.org/ $10.3390 /$ sym 13122340

Academic Editors: David Steigmann and Francesco Dell'Isola

Received: 27 October 2021

Accepted: 27 November 2021

Published: 6 December 2021

Publisher's Note: MDPI stays neutral with regard to jurisdictional claims in published maps and institutional affiliations.

Copyright: (C) 2021 by the author. Licensee MDPI, Basel, Switzerland. This article is an open access article distributed under the terms and conditions of the Creative Commons Attribution (CC BY) license (https:// creativecommons.org/licenses/by/ $4.0 /)$.

\begin{abstract}
This paper concerns finite elasto-plasticity of crystalline materials with micro-structural defects. We revisit the basic concepts: plastic distortion and decomposition of the plastic connection. The body is endowed with a structure of differential manifold. The plastic distortion is an incompatible diffeomorphism. The metric induced by the plastic distortion on the intermediate configuration (considered to be a differential manifold) is a key point in the theory, in defining the defects related to point defects, or extra-matter. The so-called plastic connection is metric, with plastic metric tensor expressed in terms of the plastic distortion and its adjoint. We prove an appropriate decomposition of the plastic connection, without any supposition concerning the non-metricity of plastic connection. All types of the lattice defects, dislocations, disclinations, and point defects are described in terms of the densities related to the elements that characterize the decomposition theorem for plastic connection. As a novelty, the measure of the interplay of the possible lattice defects is introduced via the Cartan torsion tensor. To justify the given definitions, the proposed measures of defects are compared to their counterparts corresponding to a classical framework of continuum mechanics. Thus, their physical meanings can be emphasized at once.
\end{abstract}

Keywords: elasto-plasticity; lattice defects; plastic distortion; plastic connection; differential manifolds

\section{Introduction}

The study concerns finite elasto-plasticity of crystalline materials (such as metals) with micro-structural defects.

The main objective of the paper is to revisit the basic concepts, the definition of plastic distortion and the decomposition of the plastic connection, and to introduce the measures of micro-structural defects (and their interplay) in terms of the elements that characterize the plastic connection, emphasized through the decomposition theorem. As a basic assumption, we consider the multiplicative decomposition of the deformation gradient into its components, the so-called plastic and elastic distortions. These plastic and elastic distortions are constitutive concepts, which cannot be reduced to their geometrical aspects (see Mandel [1], Teodosiu [2], and Cleja-Tigoiu and Soós [3]). Both components are incompatible (i.e., anholonomic) diffeomorphisms, which means that they are not differentials of certain appropriate vector fields. A non-local second-order theory allows us to define the measure of defects in terms of elements that characterize the so-called plastic connection. The defects existing inside elasto-plastic crystalline materials, such as dislocations, disclinations, and point-defects (involving micro-cracks and microvoids, which means that the material is damaged at the micro-level) are experimentally put into evidence (see Romanov [4]). The effects of defects on the material's behaviour are theoretically and numerically analyzed within different constitutive models (see, for instance Cleja-Tigoiu et al. [5,6]). The proposed models are scale sensitive and can be successfully applied to very small length scale (nano-mechanic) and to processes that occur in micro-seconds. 
There exists an extensive body of literature on modeling continuous distribution of defects. We recall that the defect theory within small strain framework (see De Wit [7], Fressengeas et al. [8,9]) includes the (elastic) incompatible strain and bend-twist tensor. de Wit [10] establishes that these defects correspond to concepts of non-metric and nonRiemannian geometry in the linear approximation (see also Kröner [11]). Different anisotropic damage tensors, among them the tensor that measures the non-metricity, were proposed in the paper [12,13] (see also the motivation given in the paper by Kröner [11]).

We recall here that the constitutive equations for elasto-plastic crystalline materials describe their dissipative and irreversible behaviour [14,15]. The behaviour of elastoplastic crystalline material is elastic relative to the plastically deformed configuration (see Teodosiu [2] and Mandel [1]). The dissipative nature of the plastic behavior is inferred in the constitutive framework via the free energy imbalance principle (see initial formulations in Gurtin [16] and other specific presentation in [17]). The evolution equations for the densities of defects as well as the variation of the plastic distortion are derived to be compatible with reduced dissipation inequality. These equations are rate dependent (in other words they are of the viscoplastic type) and generally non-local, i.e., partial differential equations (see [15]).

We use differential geometry concepts following Nakahara [18] and Lee [19]. Different applications of differential geometry to continuum mechanics can be found, for instance, in Noll [20], Wang [21], Steinmann [22], Epstein[23], Clayton [24], Yavari and Goriely [25], and Cleja-Ţigoiu and Ţigoiu[13], to cite a few.

In this paper, we start from the definition of the body viewed as a set (time-dependent) of differential manifolds, with the motion given as a set of $C^{2}$-diffeomorphisms, following Clayton [26] and Yavari and Goriely [27] The deformation gradient, F, which is, at any time, the differential of the motion function, $\chi$, acts on the tangent space of the body with the values in the tangent space of the body deformed into its configuration at time $t$. As we consider an elasto-plastic body, the definition of the plastic distortion is a key point of the models. The physical motivations of the plastic part of deformation are related to certain locally relaxation processes. The small neighborhoods of the material points from the deformed body are cut off the body, and free of the loading, they occupy different spatial positions. As the body contains defects that produce residual stress, it is not possible to restore a continuous body from these locally relaxed material neighborhoods without applying supplementary deformations, i.e., so-called elastic deformations. For any arbitrary particle of the body $\mathcal{B}$, we assume that, at least in principle, a neighborhood $\mathcal{N}_{x}$ in the deformed configuration can be cut off from the body and, by an unloading process, this $\mathcal{N}_{x}$ relaxes, while keeping existing inside defects (see Teodosiu [2] and Mandel [1]). For the sake of simplicity in writing formulae, we adopt here the definition of plastic distortion based on the existence of an intermediate configuration, denoted $\widetilde{\mathcal{B}}$, taken as a differentiable manifold, although we consider this intermediate configuration locally. Plastic distortion is an anholonomic diffeomorphism, denoted by $\mathbf{F}^{p}$, acting on the tangent space of the body in its initial configuration, with the values of the tangent space of the intermediate configuration.

As a novelty, we prove the decomposition theorem for the connection $\Gamma$ with $\mathrm{C}-$ metric property, where the metric tensor, $\mathrm{C}$, is represented in terms of an anholonomic diffeomorphism [26], denoted in the theorem by $\mathbf{F}$ and its adjoint. The connection $\Gamma$ is represented in terms of a connection $\mathcal{A}$ and a covariant third-order tensor field. The connection $\mathcal{A}$ is compatible with the anholonomic diffeomorphism $\mathbf{F}$ (i.e., Frobenius' conditions hold (see Cartan [28])). Concerning the tensor field, we found that one of its parts is associated with the inhomogeneity of the metric tensor, $\mathbf{g}$, of $\left(\mathcal{B}_{t}\right)$. The metric induced $\mathbf{g}$ (by $\mathbf{C}$ ) on $\left(\mathcal{B}_{t}\right)$, through the anholonomic diffeomorphism, $\mathbf{F}$, is not Euclidean. We mention here that this specific symmetric tensor is related to a certain non-metric property of connections. The other part (a tensor with specific skew-symmetry) is related to a one-differential form. The decomposition theorem is applied to an elasto-plastic body. More precisely, the diffeomorphism $\mathbf{F}$ is replaced by plastic distortion, the diffeomorphism $\mathbf{F}^{p}$, while the deformed 
configuration of the body at time $t, \mathcal{B}_{t}$, is replaced by the so-called intermediate (plastically deformed) configuration, labeled $\widetilde{\mathcal{B}}$. Under the mentioned hypotheses, we proved the decomposition theorem, that form was assumed in different versions by Bilby [29] and Noll [20], as well as Minagawa [30], Le and Stumpf [31], and Clayton [26]. Minagawa [30] considered a Cosserat continuum viewed as an ensemble of trihedron, i.e., composed of a point and three vectors, which change their directions and lengths when "a material piece is torn apart from a body". Non-metric connections were introduced by Krö (see [32], Minagawa [30], and De Wit [10]), accounting for extra-matter defects, such as interstitial atoms, vacancies, or other defects (see [11]).

Yavari and Gorieli [27] show that "in multiplicative plasticity one can combine the reference and "intermediate" configurations into parallelizable material manifolds". F ${ }^{p}$ is assumed to act on the local basis reference configuration and to give a local basis in the intermediate configuration. Using Cartan's moving frame, they prove that the intermediate configuration can be endowed with a so-called Weitzenböck connection, namely a connection compatible with a diffeomorphism, here with $\mathbf{F}^{p}$. In our decomposition of the plastic connection, we found again the presence of this type of connection (called crystal connection, following [31]), which is directly related to defects such as dislocations, and a part (a skew-symmetric tensor) that is related to disclinations, while the symmetric part concerns extra-matter defects. All these defects, physically described by Kleman and Fridel [33], correspond to the elements that enter the decomposition representation of the plastic connection.

The measures of the micro-structural defects are defined as third-order tensor fields, following [34], in terms of the elements that characterize the plastic connection, emphasized through the decomposition theorem. To justify the given definitions, we return to the classical framework of continuum mechanics. These densities can be rewritten in terms of second-order tensor fields, and thus comparison with the other definitions becomes possible, and their physical meanings can be emphasized at once. As a novelty, we mention the possible interplay between dislocations, disclinations, and extra-matter defects.

\section{Metric Tensor and Deformation Gradient}

We define the geometrical structure of the continuously deformed body as a part of the material geometric structure of the elasto-plastic body, in the sense adopted by Wang [21]: "the material geometric structure of a simple body is implied mathematically by these mechanical properties". In reverse context, the geometrical structure is described within a constitutive framework.

We introduce the definition of the continuous deformable body, the metric and the induced inner products by metrics, and the deformation gradient.

Definition 1. $A$ body $\mathcal{B}$ is a differential manifold with the structure defined by

1. the set of $n$-dimensional differentiable manifolds $\left\{\mathcal{B}_{t}\right\}, \forall t \in \mathbf{R}, t \geq 0$, with $\mathcal{B}_{0}=\mathcal{B}$;

2. the set of diffeomorphisms $\{\chi(\cdot, t) \mid t \in \mathbf{R}, t \geq 0\}$ of the class $C^{2}$, called the motion of the body,

$$
\chi(\cdot, t): \mathcal{B} \longrightarrow \mathcal{B}_{t}
$$

The velocity and acceleration of the particle $\mathbf{p}$, at time $t$ are defined by

$$
\frac{\partial^{k}}{\partial t^{k}} \chi(\mathbf{p}, t) \in \mathcal{T}_{\mathbf{p}}(\mathcal{B}) \quad \forall t \geq 0,
$$

for $\mathrm{k}=1$ and $\mathrm{k}=2$, respectively.

Here, the tangent spaces of the manifolds $\mathcal{B}$ and $\mathcal{B}_{t}$ at the point $\mathbf{p}$ and $\mathbf{q}=\chi(\mathbf{p}, t)$, respectively, are denoted by $\mathcal{T}_{\mathbf{p}} \mathcal{B}$, and $\mathcal{T}_{\mathbf{q}}\left(\mathcal{B}_{t}\right)$. 
The differential of the diffeomorphism $\chi(\cdot, t)$ at the point $\mathbf{p} \in \mathcal{B}$ is denoted by $\mathbf{F}(\mathbf{p}, t)$,

$$
d \chi(\mathbf{p}, t) \equiv \mathbf{F}(\mathbf{p}, t): \mathcal{T}_{\mathbf{p}}(\mathcal{B}) \longrightarrow \mathcal{T}_{\mathbf{q}}\left(\mathcal{B}_{t}\right)
$$

which is linear. $\mathbf{F}(\mathbf{p}, t)$ is called the deformation gradient.

\section{Notations}

At a point $\mathbf{p} \in \mathcal{B}$, the tangent space $\mathcal{T}_{\mathbf{p}}(\mathcal{B})$ is spanned by the basis $\frac{\partial}{\partial x^{\mu}}$, and the dual space or cotangent space $\mathcal{T}_{\mathbf{p}}^{*}(\mathcal{B})$ is spanned by the basis $d x^{\mu}$, where $x^{\mu}$ is the local coordinate on the chart $\left(\mathcal{U}_{i}, \varphi_{i}\right)$ to which $\mathbf{p}$ belongs.

At a point $\mathbf{q}=\chi(\mathbf{p}, t) \in \mathcal{B}_{t}$, the tangent space $\mathcal{T}_{\mathbf{q}}\left(\mathcal{B}_{t}\right)$ is spanned by the basis $\frac{\partial}{\partial y^{v}}$,

and the dual space or cotangent space $\mathcal{T}_{\mathbf{q}}^{*}\left(\mathcal{B}_{t}\right)$ is spanned by the basis $d y^{v}$

where $y^{\mu}$ is the local coordinate on the chart $\left(\mathcal{V}_{i}, \psi_{i}\right)$ to which $\mathbf{q}$ belongs.

$\mathcal{F}(\mathcal{B})$ - the set of all smooth functions from $\mathcal{B}$ to $\mathbf{R}$,

$\mathcal{X}(\mathcal{B})$ - the set of all smooth vector fields on $\mathcal{B}$.

Let us introduce a connection $\nabla: \mathcal{X}(\mathcal{B}) \times \mathcal{X}(\mathcal{B}) \longrightarrow \mathcal{X}(\mathcal{B})$ on $(\mathcal{B})$, with specific properties (see Nakahara [18] and Lee [19]). We use the notation $\nabla(\mathbf{U}, \mathbf{V})=\nabla_{\mathbf{U}} \mathbf{V}, \quad \forall \mathbf{U}, \mathbf{V} \in$ $\mathcal{X}(\mathcal{B})$, called the covariant derivative (or the directional derivative) of the vector field $\mathbf{V}$ along the vector field $\mathbf{U}$; these values are vector fields from $\mathcal{X}(\mathcal{B})$.

Moreover, the dual bases $\left\{d x^{k}\right\}$ and $\left\{d y^{\mu}\right\}$ of $\mathcal{T}_{\mathbf{q}}^{*}(\mathcal{B})$ and $\mathcal{T}_{\mathbf{q}}^{*}\left(\mathcal{B}_{t}\right)$, respectively, satisfy the orthogonality conditions

$$
d x^{i}\left(\frac{\partial}{\partial x^{k}}\right)=\delta_{k^{\prime}}^{i} \quad d y^{v}\left(\frac{\partial}{\partial y^{\mu}}\right)=\delta_{\mu}^{v} .
$$

We denote by $\nabla_{\mathbf{v}}$ the directional derivative along the vector $\mathbf{V}$, for $\mathbf{V} \in \mathcal{T}_{\mathbf{p}}(\mathcal{B})$, acting on the scalar field $f \in \mathcal{F}(\mathcal{B})$, as

$$
\nabla_{\mathbf{V}} f=\mathbf{V}[f]=V^{i} \frac{\partial f}{\partial x^{i}}
$$

and on the tensor fields of arbitrary type, say $\mathbf{T}_{1}$ and $\mathbf{T}_{2}$, as

$$
\nabla_{\mathbf{V}}\left(\mathbf{T}_{1} \otimes \mathbf{T}_{2}\right)=\nabla_{\mathbf{V}}\left(\mathbf{T}_{1}\right) \otimes \mathbf{T}_{2}+\mathbf{T}_{1} \otimes\left(\nabla_{\mathbf{V}} \mathbf{T}_{2}\right)
$$

where $\mathbf{T}_{1}$ and $\mathbf{T}_{2}$ are tensor fields of arbitrary type.

\subsection{Metric and Induced Inner Product}

We now introduce the metric.

Assumption 1. $\mathcal{B}$ is endowed with a metric expressed as

$$
\begin{aligned}
& \mathbf{C}(\mathbf{p}, t)=C_{i j}(\mathbf{p}, t) d x^{i} \otimes d x^{j}, \quad C_{i j}(\mathbf{p}, t)=\mathbf{C}(\mathbf{p}, t)\left(\frac{\partial}{\partial x^{i}}, \frac{\partial}{\partial x^{j}}\right), \\
& \text { or in component representation } \quad \mathbf{C}(\mathbf{p}, t)(\mathbf{U}, \mathbf{V})=C_{i j}(\mathbf{p}, t) U^{i} V^{j}, \quad \forall \mathbf{U}, \mathbf{V} \in \mathcal{T}_{\mathbf{p}}(\mathcal{B}),
\end{aligned}
$$

with the matrix $\left(\mathbf{C}_{i j}(\mathbf{p}, t)\right)$ symmetric and positive definite.

Remark 1. The metric, $\mathbf{C}(\mathbf{p}, t)$, can be viewed as a tensor:

$$
\begin{aligned}
& \mathbf{C}(\mathbf{p}, t): \mathcal{T}_{\mathbf{p}}(\mathcal{B}) \longrightarrow \mathcal{T}_{\mathbf{p}}^{*}(\mathcal{B}), \text { linear, i.e., } \\
& \mathbf{C}(\mathbf{p}, t) \mathbf{V}=C_{i j}(\mathbf{p}, t) V^{j} d x^{i}, \quad \forall \quad \mathbf{V} \in \mathcal{T}_{\mathbf{p}}(\mathcal{B}), \\
& \Longrightarrow(\mathbf{C}(\mathbf{p}, t) \mathbf{V}) \mathbf{U}=\mathbf{C}(\mathbf{p}, t)(\mathbf{U}, \mathbf{V})=C_{i j}(\mathbf{p}, t) V^{j} U^{i}, \forall \mathbf{U} \in \mathcal{T}_{\mathbf{p}}(\mathcal{B}),
\end{aligned}
$$


where $\mathbf{U}=U^{j} \frac{\partial}{\partial x^{j}}$ and $\mathbf{V}=V^{j} \frac{\partial}{\partial x^{j}} \in \mathcal{T}_{\mathbf{p}}(\mathcal{B})$.

Remark 2. As $\chi(\cdot, t): \mathcal{B} \longrightarrow \mathcal{B}_{t}$ is a diffeomorphism from a differentiable manifold with a metric, $C(\mathbf{p}, t)$, the induced natural metric, denoted by $g(\mathbf{q}, t)$, can be introduced on $\mathcal{B}_{t}$, namely

$$
\mathbf{g}(\mathbf{q}, t)=g_{i j}(\mathbf{q}, t) d y^{i} \otimes d y^{j}, \quad \text { with } \quad g_{i j}(\mathbf{q}, t)=C_{k l}(\mathbf{p}, t) \frac{\partial x^{k}}{\partial y^{i}} \frac{\partial x^{l}}{\partial y^{j}},
$$

in component representation. Here, $\mathbf{q}=\chi(\mathbf{p}, t)$.

We now introduce the inner product induced by the metric, between any two vectors or two dual vectors. Moreover, the inner product is extended to define an inner product between a vector and a dual vector, which is compatible with Nakahara's definition in [18].

As there exists a metric, the inner product can be extended to define the product between any two vectors.

Nakahara, in [18], defined the inner product between a vector and a dual vector as

$$
\begin{gathered}
a . \quad<,>: \mathcal{T}_{\mathbf{p}}^{*}(\mathcal{B}) \times \mathcal{T}_{\mathbf{p}}(\mathcal{B}) \longrightarrow \mathbf{R}, \quad<\omega, \mathbf{U}>=\omega(\mathbf{U}), \\
<\omega, \mathbf{U}>=\omega_{\nu} U^{\mu}\left(d x^{\nu}\left(\frac{\partial}{\partial x^{\mu}}\right)\right)=\omega_{\mu} U^{\mu}, \\
\forall \omega=\omega_{v} d x^{\nu} \in \mathcal{T}_{\mathbf{p}}^{*}(\mathcal{B}), \quad \mathbf{U}=U^{\mu} \frac{\partial}{\partial x^{\mu}} \in \mathcal{T}_{\mathbf{p}}(\mathcal{B}),
\end{gathered}
$$

as a consequence of (4). As the symmetry property $\langle\mathbf{U}, \omega\rangle$ is not yet defined, there is a so-called duality product and not an inner product.

Proposition 1. The pairs $(\omega, \mathbf{V}) \in \mathcal{T}_{\mathbf{p}}^{*}(\mathcal{B}) \times \mathcal{T}_{\mathbf{p}}(\mathcal{B})$ and $(\mathbf{U}, \mathbf{V}), \in \mathcal{T}_{\mathbf{p}}(\mathcal{B}) \times \mathcal{T}_{\mathbf{p}}(\mathcal{B})$, respectively, are related (via the metric) in such a way as to have the equality

$$
\begin{aligned}
& \omega_{\mu} V^{\mu}=\mathbf{C}_{i j}(\mathbf{p}, t) U^{j} V^{i}, \forall \mathbf{V} \in \mathcal{T}_{\mathbf{p}}(\mathcal{B}) \\
& \text { where } \omega=\omega_{v} d x^{v} \in \mathcal{T}_{\mathbf{p}}^{*}(\mathcal{B}), \quad \mathbf{U}=U^{\mu} \frac{\partial}{\partial x^{\mu}}, \mathbf{V}=V^{i} \frac{\partial}{\partial x^{i}} \in \mathcal{T}_{\mathbf{p}}(\mathcal{B}) .
\end{aligned}
$$

For any $\mathbf{U} \in \mathcal{T}_{\mathbf{p}}(\mathcal{B})$, there exists $\omega \in \mathcal{T}_{\mathbf{q}}^{*}(\mathcal{B})$, such that

$$
\omega=\omega_{i} d x^{i}, \quad \omega_{i}=C_{i j} U^{j}, \quad \omega_{\mu} V^{\mu}=C_{i j}(\mathbf{p}, t) U^{j} V^{i} .
$$

For any $\omega \in \mathcal{T}_{\mathbf{p}}^{*}(\mathcal{B})$, there exists $\mathbf{U} \in \mathcal{T}_{\mathbf{p}}(\mathcal{B})$, such that

$$
U^{j}=C^{k j}(\mathbf{p}, t) \omega_{k}, \quad C_{i j}(\mathbf{p}, t) U^{j} V^{i}=\omega_{k} V^{k}, \forall \mathbf{V} \in \mathcal{T}_{\mathbf{p}}(\mathcal{B}),
$$

where the matrix $\left(C^{i j}(\mathbf{p}, t)\right)$ is the inverse matrix $\left(C_{i j}(\mathbf{p}, t)\right)^{-1}$.

Thus, the following inner product between a vector and a dual vector is well defined

$$
\begin{aligned}
& \text { b. } \quad\left\langle\omega, \mathbf{V}>=\mathbf{C}(\mathbf{p}, t)(\mathbf{V}, \mathbf{U}), \forall \mathbf{V} \in \mathcal{T}_{\mathbf{p}}(\mathcal{B}), \forall \omega \in \mathcal{T}_{\mathbf{p}}^{*}(\mathcal{B}),\right. \\
& \text { where } \mathbf{U}=U^{j} \frac{\partial}{\partial x^{j}}, U^{j}=C^{j k} \omega_{k} .
\end{aligned}
$$

Obviously, the inner product defined by b. is compatible with (10). 
The inner product is also defined between the elements of the same spaces in the case of differentiable manifolds with a metric, as follows:

$$
\begin{aligned}
\text { c. } \quad<, & >: \mathcal{T}_{\mathbf{p}}(\mathcal{B}) \times \mathcal{T}_{\mathbf{p}}(\mathcal{B}) \longrightarrow \mathbf{R}, \\
& <\mathbf{U}, \mathbf{V}>=\mathbf{C}(\mathbf{U}, \mathbf{V}), \quad \text { i.e., } \quad<\mathbf{U}, \mathbf{V}>=C_{i j} U^{i} V^{j} \quad \mathbf{U}, \mathbf{V} \in \mathcal{T}_{\mathbf{p}}(\mathcal{B}), \\
\text { d. } \quad<, & >: \mathcal{T}_{\mathbf{p}}^{*}(\mathcal{B}) \times \mathcal{T}_{\mathbf{p}}^{*}(\mathcal{B}) \longrightarrow \mathbf{R}, \\
& <\omega, \zeta>=\omega_{i} C^{i j} \zeta_{j}, \quad \omega=\omega_{k} d x^{k}, \zeta=\zeta_{j} d x^{j} \in \mathcal{T}_{\mathbf{p}}^{*}(\mathcal{B}) .
\end{aligned}
$$

Remark 3. For the sake of simplicity, we do not introduce different notations (say, to mention the metric) for the above inner products if it is not necessary.

Proposition 2. The metric $\mathbf{C}(\mathbf{p}, t)$ gives rise to an isomorphism between $\mathcal{T}_{\mathbf{p}} \mathcal{B}$ and $\mathcal{T}_{\mathbf{p}}^{*} \mathcal{B}$.

Proof. As a consequence of the metric property of the differentiable manifold, via the relationships (8) and (13), we proved that, for all $\mathbf{U} \in \mathcal{T}_{\mathbf{p}}(\mathcal{B})$, there exists $\omega \in \mathcal{T}_{\mathbf{p}}^{*}(\mathcal{B})$, and vice versa. Moreover, $\langle\omega, \omega\rangle=<\mathbf{U}, \mathbf{U}\rangle$.

\subsection{Deformation Gradient and Its Adjoint}

Proposition 3. The deformation gradient is given by the following representation:

$$
\mathbf{F}(\mathbf{p}, t): \mathcal{T}_{\mathbf{p}}(\mathcal{B}) \longrightarrow \mathcal{T}_{\mathbf{q}}\left(\mathcal{B}_{t}\right), \quad \mathbf{F}(\mathbf{p}, t)=F^{i}{ }_{j} \frac{\partial}{\partial y^{i}} \otimes d x^{j},
$$

while the adjoint of the deformation tensor, $\mathbf{F}^{*}(\mathbf{p}, t)$, is given by

$$
\mathbf{F}^{*}(\mathbf{p}, t): \mathcal{T}_{\mathbf{q}}^{*}\left(\mathcal{B}_{t}\right) \longrightarrow \mathcal{T}_{\mathbf{p}}^{*}(\mathcal{B}), \quad \mathbf{F}^{*}(\mathbf{p}, t)=F^{k}{ }_{s} d x^{\mathcal{S}} \otimes \frac{\partial}{\partial y^{k}}
$$

Proof. We apply the definition of the adjoint of a tensor. Let $\mathbf{A}: \mathcal{M} \longrightarrow \mathcal{N}$ be a linear map, where $\mathcal{M}$ and $\mathcal{N}$ are differentiable manifolds, having dual manifolds denoted by $\mathcal{M}^{*}$ and $\mathcal{N}^{*}$. The rule to define the adjoint $\mathbf{A}^{*}: \mathcal{N}^{*} \longrightarrow \mathcal{M}^{*}$ is given by

$$
\mathbf{A}^{*}(\omega) \mathbf{V}=<\omega, \mathbf{A V}>, \quad \forall \omega \in \mathcal{N}^{*}, \quad \forall \mathbf{V} \in \mathcal{M}
$$

Following the rule (18), written for $\mathbf{F}(\mathbf{p}, t): \mathcal{T}_{\mathbf{p}} \mathcal{B} \longrightarrow \mathcal{T}_{\mathbf{q}} \mathcal{B}_{t}$, which is linear, we derive the equalities

$$
\begin{aligned}
& \mathbf{F}^{*}(\mathbf{p}, t)(\omega) \mathbf{V}=\omega_{k}<\mathbf{F}^{*}(\mathbf{p}, t)\left(d y^{k}\right), \mathbf{V}>=\omega_{k} V^{s}<\mathbf{F}^{*}(\mathbf{p}, t)\left(d y^{k}\right), \frac{\partial}{\partial x^{s}}>= \\
& =<\omega, \mathbf{F}(\mathbf{p}, t) \mathbf{V}>=\omega_{k} V^{s} F_{s,}^{k}
\end{aligned}
$$

written $\quad \forall \quad \omega=\omega_{k} d y^{k} \in \mathcal{T}_{\mathbf{q}}^{*} \mathcal{B}_{t}$, and $\quad \forall \quad \mathbf{V}=V^{s} \frac{\partial}{\partial x^{s}} \in \mathcal{T}_{\mathbf{p}} \mathcal{B}$.

Thus, from the equality

$$
<\mathbf{F}^{*}(\mathbf{p}, t)\left(d y^{k}\right), \frac{\partial}{\partial x^{s}}>=<d y^{k}, \mathbf{F}(\mathbf{p}, t) \frac{\partial}{\partial x^{s}}>=F_{s}^{k}(\mathbf{p}, t),
$$

Formula (17) results. 
Remark 4. The rules ((15)c.) applied to the basis vectors in $\mathcal{T}_{\mathbf{q}} \mathcal{B}_{t}$ and in $\mathcal{T}_{\mathbf{p}} \mathcal{B}$, respectively, give rise to the scalars

$$
<\frac{\partial}{\partial y^{k}}, \frac{\partial}{\partial y^{i}}>=g_{k i}, \quad<\frac{\partial}{\partial x^{k}}, \frac{\partial}{\partial x^{i}}>=C_{k i}
$$

while the rule (14) acts on the basis vectors taken from the tangent space $\mathcal{T}_{\mathbf{q}} \mathcal{B}_{t}$ and its dual $\mathcal{T}_{\mathbf{q}}^{*} \mathcal{B}_{t}$ as

$$
<\frac{\partial}{\partial y^{k}}, d y^{i}>=\delta^{i}{ }_{k}
$$

Remark 5. Let us consider the tensors $\mathbf{A} \in \mathcal{T}_{2}{ }^{0}(\mathcal{B}), \mathbf{B} \in \mathcal{T}_{0}{ }^{2}(\mathcal{B}), \mathbf{E} \in \mathcal{T}_{1}^{1}(\mathcal{B}), \mathbf{D} \in \mathcal{T}^{1}{ }_{1}(\mathcal{B})$

$$
\begin{aligned}
& \mathbf{A}=A_{i j} d x^{i} \otimes d x^{j}, \quad \mathbf{B}=B^{k l} \frac{\partial}{\partial x^{k}} \otimes \frac{\partial}{\partial x^{l}}, \\
& \mathbf{E}=E_{m}{ }^{n} d x^{m} \otimes \frac{\partial}{\partial x^{n}}, \quad \mathbf{D}=D^{s}{ }_{t} \frac{\partial}{\partial x^{s}} \otimes d x^{t} .
\end{aligned}
$$

Based on the inner product (see Formulas (21) and (22)), the following composition rules concerning the tensors are introduced:

$$
\begin{aligned}
& \mathbf{A} \circ \mathbf{B}=A_{i j} B^{k l} d x^{i} \otimes<d x^{j}, \frac{\partial}{\partial x^{k}}>\frac{\partial}{\partial x^{l}}=A_{i j} B^{j l} d x^{i} \otimes \frac{\partial}{\partial x^{l}}, \\
& \mathbf{A} \circ \mathbf{E}=A_{i j} E_{m}{ }^{n} d x^{i} \otimes<d x^{j}, d x^{m}>\frac{\partial}{\partial x^{n}}=A_{i j} C^{j m} E_{m}{ }^{n} d x^{i} \otimes \frac{\partial}{\partial x^{n}}, \\
& \mathbf{E} \circ \mathbf{D}=E_{m}{ }^{n} D^{s}{ }_{t} d x^{m} \otimes<\frac{\partial}{\partial x^{n}}, \frac{\partial}{\partial x^{s}}>d x^{t}=E_{m}{ }^{n} C_{n s} D^{s}{ }_{t} d x^{m} \otimes d x^{t},
\end{aligned}
$$

and so on. In the two last compositions, the presence of the components of the matrices associated with the metric tensor can be observed.

Assumption 2. The metric tensor $\mathbf{C}(\mathbf{p}, t)$ and the deformation gradient $\mathbf{F}(\mathbf{p}, t)$, for any $\mathbf{p} \in \mathcal{B}$ are related by

$$
\mathbf{C}(\mathbf{p}, t)=\mathbf{F}^{*}(\mathbf{p}, t) \circ \mathbf{F}(\mathbf{p}, t) .
$$

The component representation Formula (25) follows

$$
\begin{aligned}
& F_{m}^{i}(\mathbf{p}, t) g_{i s}(\mathbf{q}, t) F^{s}{ }_{n}(\mathbf{p}, t)=C_{m n}(\mathbf{p}, t), o r \\
& g_{m n}(\mathbf{q}, t)=\left(F^{-1}\right)^{i}{ }_{m}(\mathbf{p}, t) C_{i j}(\mathbf{p}, t)\left(F^{-1}\right)^{j}{ }_{n},
\end{aligned}
$$

as a consequence of the Formulas (16) and (17), together with the composition rule as in (24).

Traditionally, in continuum mechanics, the right Cauchy-Green tensor $\mathbf{C}$ is defined by $\mathbf{C}=\mathbf{F}^{T} \mathbf{F}$, being associated with a given deformation gradient $\mathbf{F}$. Following Yavari and Goriely [25], we consider the right Cauchy-Green tensor, denoted here as $\mathbf{C}^{(G)}$ to avoid confusion with the previously introduced formulae, to be defined by

$$
\mathbf{C}^{(G)}(\mathbf{p}, t): \mathcal{T}_{\mathbf{p}}(\mathcal{B}) \longrightarrow \mathcal{T}_{\mathbf{p}}(\mathcal{B}), \quad \text { linear, } \quad \mathbf{C}^{(G)}=\mathbf{F}^{T} \mathbf{F}
$$


Here, the deformation gradient $\mathbf{F}$ is arbitrarily given as $\mathbf{F}: \mathcal{T}_{\mathbf{p}} \mathcal{B} \longrightarrow \mathcal{T}_{\mathbf{q}} \mathcal{B}_{t}$, when the differential manifolds $\mathcal{B}$ and $\mathcal{B}_{t}$ with the metrics $\mathbf{G}$ and $\mathbf{g}$, respectively, are considered. The transpose of the deformation gradient is introduced as

$$
\begin{aligned}
& \mathbf{F}^{T}: \mathcal{T}_{\mathbf{q}} \mathcal{B}_{t} \longrightarrow \mathcal{T}_{\mathbf{p}} \mathcal{B}, \quad \text { such that } \\
& <\mathbf{F V}, \mathbf{v}>_{\mathbf{g}}=<\mathbf{V}, \mathbf{F}^{T} \mathbf{v}>_{\mathbf{G}} \forall \mathbf{V} \in \mathcal{T}_{\mathbf{p}} \mathcal{B}, \forall \mathbf{v} \in \mathcal{T}_{\mathbf{q}} \mathcal{B}_{t}
\end{aligned}
$$

The relations are written in our notations, while mentioning the appropriate metrics. In the component, the Formulas (28) and (27) become

$$
\begin{aligned}
& \left(F^{T}(\mathbf{q}, t)\right)_{a}^{A}=g_{a b}(\mathbf{q}, t) F_{B}^{b}(\mathbf{p}, t) G^{A B}(\mathbf{p}, t), \text { and } \\
& \left(\mathbf{C}^{(G)}\right)^{A}{ }_{B}=\left(F^{T}\right)^{A}{ }_{a} F^{a}{ }_{B}=G^{A Q}(\mathbf{p}, t) F_{B}^{a} F_{Q}^{b}(\mathbf{p}, t) g_{a b}(\mathbf{q}, t), \quad \text { or } \\
& \left(\mathbf{C}^{(G)}\right)_{A B}=F_{A}^{a} F_{B}^{b} g_{a b},
\end{aligned}
$$

where $\left\{g_{a b}\right\}$ and $\left\{G^{A B}\right\}$ are components of the metric tensors. The last formula in (29) is derived by lowering the indices using the metric $\mathbf{G}$.

Remark 6. We give the arguments that justify our representation (25) for the metric tensor $\mathbf{C}$ in terms of $\mathbf{F}$ and its adjoint $\mathbf{F}^{*}$.

1. Let us remark that $\left(\mathbf{C}^{(G)}\right)_{A B} \equiv G_{A B}, \quad \forall A, B=1,2,3$, (which means that the components of the Cauchy-Green tensor are the metric components) if and only if

$$
G_{A B}=F_{A}^{a} F_{B}^{b} g_{a b}
$$

Thus, the metric components of the differential manifolds are related by the components of the deformation gradient, considered $\mathbf{F}$.

2. By definition of the Cauchy-Green tensor, (27), $\mathbf{C}^{(G)}(\mathbf{p}, t) \mathbf{U} \in \mathcal{T}_{\mathbf{p}}(\mathcal{B}) \quad \forall \mathbf{U} \in \mathcal{T}_{\mathbf{p}}(\mathcal{B})$, while the metric tensor, denoted here by $\mathbf{G}$, applied to a vector field is a dual vector, namely $\mathbf{G}(\mathbf{p}, t) \mathbf{U} \in \mathcal{T}_{\mathbf{p}}^{*}(\mathcal{B})$, as it follows from (8). Thus, only if $\mathcal{T}_{\mathbf{p}}^{*}(\mathcal{B})$ is identified with $\mathcal{T}_{\mathbf{p}}(\mathcal{B})$ can we say that a metric tensor can be viewed as a Cauchy-Green tensor. Moreover, from (30) the metric tensor with the components $\left\{G_{A B}\right\}$ induces the metric with the components $\left\{g_{a b}(\mathbf{q}, t)\right\}$ by the deformation gradient, represented in terms of its components $F^{a} A$.

3. Formula (29) ${ }_{1}$ expresses the components of the transpose tensor in terms of both metrics, while Formula (26) gives rise to the induced metric $\left\{g_{\text {is }}\right\}$ on $\mathcal{B}_{t}$ by $\mathbf{F}$.

4. In classical continuum mechanics, all vector fields refer to the same vector space, $\mathcal{V}$, which is associated with the Euclidean space $\mathcal{E}$. The dual space is identified with the vector space. Consequently, the adjoint and the transpose of a tensor coincide. Moreover, the metric and non-metric properties are independent feature of the connection.

5. All the formulas derived above remain valid if the deformation gradient is replaced by an anholonomic diffeomorphism.

\section{Material Connection-Revisited Decomposition Theorem}

Take a chart $(\mathcal{U}, \varphi)$, with the coordinate $\left\{x^{k}\right\}$ on $\mathcal{B}$ and define functions $\Gamma^{s}{ }_{k j}, \quad s, k, j \in$ $\{1,2,3\}$, called connection coefficients by

$$
\begin{aligned}
& \nabla_{k} \frac{\partial}{\partial x^{j}}=\Gamma_{k j}^{s} \frac{\partial}{\partial x^{s}}, \quad \nabla_{k}\left(d x^{j}\right)=-\Gamma_{k s}^{j} d x^{s}, \\
& \text { with the notation } \quad \nabla_{k} \equiv{\frac{\partial}{\partial x^{k}}}^{\prime}
\end{aligned}
$$


for the covariant (or directional) derivative along the vectors $\left\{\frac{\partial}{\partial x^{j}}\right\}$, which forms a basis in $\mathcal{T}_{\mathbf{p}}(\mathcal{B})$. Here, $\left\{d x^{s}\right\}$ is the basis in $\mathcal{T}_{\mathbf{p}}^{*}(\mathcal{B})$.

Let us introduce the notation $\nabla_{\Gamma}$ for the connection $\nabla$ with the connection coefficients $\boldsymbol{\Gamma}$, given by Formula (31).

Remark 7. Given the coefficient connection $\left\{\Gamma^{s}{ }_{k j}\right\}$, we propose a tensor-type formulae

$$
\Gamma=\Gamma^{s}{ }_{k j} \frac{\partial}{\partial x^{m}} \otimes d x^{j} \otimes d x^{k},
$$

to emphasize the coordinate basis in which the coefficients are computed.

\subsection{Covariant Derivatives of the Tensor Fields}

Definition 2. Let a tensor field on $\mathcal{B}$ at time tbe given as an element of $\mathcal{T}^{q}{ }_{r}(\mathcal{B})$, namely the tensor field $\mathbf{p} \in \mathcal{B} \longrightarrow \mathbf{T}(\mathbf{p}, t) \in \mathcal{T}^{q}{ }_{r}(\mathcal{B})$, which is given in components by

$$
\mathbf{T}(\mathbf{p}, t)=T^{i_{1} \ldots i_{q}}{ }_{j_{1} \ldots j_{r}} \frac{\partial}{\partial x^{i_{1}}} \otimes \cdots \otimes \frac{\partial}{\partial x^{i_{q}}} \otimes d x^{j_{1}} \otimes \cdots \otimes d x^{j_{r}} .
$$

The covariant derivative of $\mathbf{T}(\cdot, t)$ at $\mathbf{p} \in \mathcal{B}$ is a tensor field, denoted by $\nabla_{k} \mathbf{T}(\mathbf{p}, t) \in \mathcal{T}^{q}{ }_{r}(\mathcal{B})$ and computed following the rule written in coordinate charts as

$$
\begin{aligned}
& \nabla_{k} \mathbf{T}(\mathbf{p}, t)=\frac{\partial}{\partial x^{k}}\left(T^{i_{1} \ldots i_{q}}{ }_{j_{1} \ldots j_{r}}\right) \frac{\partial}{\partial x^{i_{1}}} \otimes \cdots \otimes \frac{\partial}{\partial x^{i_{q}}} \otimes d x^{j_{1}} \otimes \cdots \otimes d x^{j_{r}}+ \\
& +T^{i_{1} \ldots \ldots i_{q}}{ }_{j_{1} \ldots j_{r}} \frac{\partial}{\partial x^{i_{1}}} \otimes \cdots \otimes \Gamma_{k i_{s}}^{i_{m}} \frac{\partial}{\partial x^{i_{m}}} \otimes \cdots \otimes \frac{\partial}{\partial x^{q}} \otimes d x^{j_{1}} \otimes \cdots \otimes d x^{j_{r}}- \\
& -T^{i_{1} \ldots i_{s} \ldots i_{q}}{ }_{j_{1} \ldots j_{s} \ldots j_{r}} \frac{\partial}{\partial x^{i_{1}}} \otimes \cdots \otimes \frac{\partial}{\partial x^{q}} \otimes d x^{j_{1}} \otimes \cdots \otimes \Gamma_{k j_{m}}^{j_{s}} d x^{j_{m}} \otimes \cdots \otimes d x^{j_{r}} .
\end{aligned}
$$

The above tensor notations are similar to those used by Clayton [26].

Assumption 3. The connection $\nabla_{\Gamma}$ is metric.

Definition 3. The connection $\nabla_{\boldsymbol{\Gamma}}$ is metric if the covariant derivative of the metric tensor $\mathbf{C}(\mathbf{p}, t)$ is vanishing, namely $\nabla_{k} \mathbf{C}(\mathbf{p}, t)=0, \forall k$

$$
\nabla_{k} \mathbf{C}(\mathbf{p}, t) \equiv\left\{\frac{\partial}{\partial x^{k}} C_{n j}-C_{s j} \Gamma^{s}{ }_{k n}-C_{n s} \Gamma^{s}{ }_{k j}\right\} d x^{n} \otimes d x^{j} \equiv 0 .
$$

Proposition 4. The covariant derivative of the field $\mathbf{F}$ is computed by

$$
\nabla_{k} \mathbf{F}(\mathbf{p}, t)=\frac{\partial}{\partial x^{k}}\left(F^{i}{ }_{j}\right) \frac{\partial}{\partial y^{i}} \otimes d x^{j}+F^{i}{ }_{j} \nabla_{k}\left(\frac{\partial}{\partial y^{i}}\right) \otimes d x^{j}-F_{j}^{i} \Gamma_{k s}^{j} \frac{\partial}{\partial y^{i}} \otimes d x^{s} .
$$

The covariant derivative of the field $\mathbf{F}^{*}$ is given by

$$
\begin{aligned}
\nabla_{k} \mathbf{F}^{*}(\mathbf{p}, t) & =\frac{\partial}{\partial x^{k}}\left(F^{s}{ }_{n}\right) d x^{n} \otimes \frac{\partial}{\partial y^{s}}- \\
& -F^{s}{ }_{n} \Gamma^{n}{ }_{k l} d x^{l} \otimes \frac{\partial}{\partial y^{s}}+F^{s}{ }_{n} d x^{n} \otimes \nabla_{k}\left(\frac{\partial}{\partial y^{s}}\right) .
\end{aligned}
$$


We compose Formulas (37) and (33) with (16) and (17), respectively, by applying the rules similar to those listed in (24) together with (21) and (22). We provide the formulas

$$
\begin{gathered}
\nabla_{k} \mathbf{F}^{*} \circ \mathbf{F}=F^{i}{ }_{j} \frac{\partial}{\partial x^{k}}\left(F^{s}{ }_{n}\right) g_{s i} d x^{n} \otimes d x^{j}-F^{s}{ }_{l} F^{i}{ }_{j} \Gamma^{l}{ }_{k n} g_{s i} d x^{n} \otimes d x^{j}+ \\
+F^{s}{ }_{n} F^{i}{ }_{j}<\nabla_{k}\left(\frac{\partial}{\partial y^{s}}\right), \frac{\partial}{\partial y^{i}}>d x^{n} \otimes d x^{j} . \\
\mathbf{F}^{*} \circ \nabla_{k} \mathbf{F}=F^{s}{ }_{n} \frac{\partial}{\partial x^{k}}\left(F^{i}{ }_{j}\right) g_{s i} d x^{n} \otimes d x^{j}+ \\
+F^{s}{ }_{n} F^{i}{ }_{j} d x^{n} \otimes<\frac{\partial}{\partial y^{s}}, \nabla_{k}\left(\frac{\partial}{\partial y^{i}}\right)>d x^{j}-F^{s}{ }_{n} F^{i}{ }_{l} g_{s i} \Gamma^{l}{ }_{k j} d x^{n} \otimes d x^{j} .
\end{gathered}
$$

We now introduce a connection to the body manifold $\mathcal{B}$, denoted by $\mathcal{A}$, which is compatible with the tensor field $\mathbf{F}$ and allows a coordinate representation of

$$
\begin{aligned}
\mathcal{A}(\mathbf{p}, t) & =\left((\mathbf{F}(\mathbf{p}, t))^{-1}\right)^{m}{ }_{s} \frac{\partial}{\partial x^{k}}\left(F^{s}{ }_{j}(\mathbf{p}, t)\right) \frac{\partial}{\partial x^{m}} \otimes d x^{j} \otimes d x^{k} \equiv \\
& \equiv \mathcal{A}^{m}{ }_{k j} \frac{\partial}{\partial x^{m}} \otimes d x^{j} \otimes d x^{k} .
\end{aligned}
$$

By composing the metric tensor $\mathbf{C}(\mathbf{p}, t)$, given by (7), with (39), we provide the equalities

$$
\begin{aligned}
& \mathbf{C}(\mathbf{p}, t) \circ \mathcal{A}(\mathbf{p}, t)= \\
& \left.=\left(C_{q n}(\mathbf{p}, t) d x^{q} \otimes d x^{n}\right) \circ\left((\mathbf{F}(\mathbf{p}, t))^{-1}\right)^{m}{ }_{s} \frac{\partial}{\partial x^{k}} F^{s}{ }_{j}(\mathbf{p}, t)\right) \frac{\partial}{\partial x^{m}} \otimes d x^{j} \otimes d x^{k}= \\
& \left.=C_{q m}(\mathbf{p}, t)\left((\mathbf{F}(\mathbf{p}, t))^{-1}\right)^{m}{ }_{s} \frac{\partial}{\partial x^{k}} F_{j}^{s}(\mathbf{p}, t)\right) d x^{q} \otimes d x^{m} \otimes d x^{k}= \\
& \left.=F^{l}{ }_{q}(\mathbf{p}, t) g_{l u}(\mathbf{p}, t) F^{u}{ }_{m}(\mathbf{p}, t)\left((\mathbf{F}(\mathbf{p}, t))^{-1}\right)^{m}{ }_{s} \frac{\partial}{\partial x^{k}} F^{s}{ }_{j}(\mathbf{p}, t)\right) d x^{q} \otimes d x^{j} \otimes d x^{k},
\end{aligned}
$$

if we account for (26). Finally, Formula (41) becomes

$$
\mathrm{C} \circ \mathcal{A}=F^{i}{ }_{j} \frac{\partial}{\partial x^{k}}\left(F^{s}{ }_{n}\right) g_{s i} d x^{n} \otimes d x^{j} \otimes d x^{k} \equiv C_{n m} \mathcal{A}^{m}{ }_{k j} d x^{n} \otimes d x^{j} \otimes d x^{k},
$$

where the connection coefficients $\mathcal{A}^{m}{ }_{k j}$ are written in (40).

Proposition 5. Formulas (38) and (39), respectively, can be written as

$$
\begin{aligned}
\nabla_{k} \mathbf{F}^{*} \circ \mathbf{F} & =C_{j m}\left(\mathcal{A}_{k n}^{m}-\Gamma^{m}{ }_{k n}\right) d x^{n} \otimes d x^{j}+ \\
& +F^{s}{ }_{n} F^{i}{ }_{j}<\nabla_{k}\left(\frac{\partial}{\partial y^{s}}\right), \frac{\partial}{\partial y^{i}}>d x^{n} \otimes d x^{j}
\end{aligned}
$$

and

$$
\begin{aligned}
\mathbf{F}^{*} \circ \nabla_{k} \mathbf{F} & =C_{n m}\left(\mathcal{A}^{m}{ }_{k j}-\Gamma^{m}{ }_{k j}\right) d x^{n} \otimes d x^{j}+ \\
& +F^{s}{ }_{n} F^{i}{ }_{j}<\frac{\partial}{\partial y^{s}}, \nabla_{k}\left(\frac{\partial}{\partial y^{i}}\right)>d x^{n} \otimes d x^{j},
\end{aligned}
$$

if we take into account Formula (42). 
We evaluate the following tensor and scalar fields, respectively, when the directional derivative along the vector basis defined by (31) is applied:

$$
\begin{aligned}
& \nabla_{k}\left(\frac{\partial}{\partial y^{s}}\right) \equiv \nabla_{\frac{\partial}{\partial x^{k}}}\left(\frac{\partial}{\partial y^{s}}\right) \text { for } \frac{\partial}{\partial y^{s}} \in \mathcal{T}_{\mathbf{q}}\left(\mathcal{B}_{t}\right), \text { and } \\
& \nabla_{k}\left(\frac{\partial}{\partial y^{s}}\right) \circ \frac{\partial}{\partial y^{i}} \equiv<\nabla_{k}\left(\frac{\partial}{\partial y^{s}}\right), \frac{\partial}{\partial y^{i}}>,
\end{aligned}
$$

as can be seen in the following proposition:

Proposition 6. Under the hypothesis that the connection $\nabla_{\Gamma}$ is metric, there exist scalar fields $\left\{\widetilde{Q}_{k s i}\right\}$, such that

$$
\nabla_{k}\left(\frac{\partial}{\partial y^{s}}\right) \circ \frac{\partial}{\partial y^{i}}+\frac{\partial}{\partial y^{i}} \circ \nabla_{k}\left(\frac{\partial}{\partial y^{s}}\right)=2 \widetilde{Q}_{k s i}
$$

which are symmetric, i.e., $\quad \widetilde{Q}_{k s i}=\widetilde{Q}_{k i s}, \quad \forall k, i, s$.

Moreover, the scalars $Q_{k n j}$

$$
Q_{k n j}=F_{n}^{s} F_{j}^{i} \widetilde{Q}_{k s i}, \quad \text { have the same symmetry } Q_{k n j}=Q_{k j n}, \quad \forall k, j, n .
$$

Proof. By the map $\chi(\cdot, t)$, the tangent field $\left\{\frac{\partial}{\partial x^{s}}\right\}$ induces the tangent field $\left\{\frac{\partial}{\partial y^{s}}\right\}$, namely

$$
\frac{\partial}{\partial y^{s}}=V^{l} \frac{\partial}{\partial x^{l}}
$$

where $\left(V^{l}{ }_{s}\right)$ denotes the Jacobian matrix. We applied (31) to (48):

$$
\begin{aligned}
& \nabla_{k}\left(\frac{\partial}{\partial y^{s}}\right)=\frac{\partial}{\partial x^{k}}\left(V_{s}^{l}\right) \frac{\partial}{\partial x^{l}}+V_{s}^{l} \Gamma^{q}{ }_{k l} \frac{\partial}{\partial x^{q}}, \text { and } \\
& \nabla_{k}\left(\frac{\partial}{\partial y^{s}}\right) \circ V^{m}{ }_{i} \frac{\partial}{\partial x^{m}}=\frac{\partial}{\partial x^{k}}\left(V_{s}^{l}\right) V^{m}{ }_{i} C_{l m}+V_{s}^{l} V^{m}{ }_{i} \Gamma^{q}{ }_{k l} C_{m q} .
\end{aligned}
$$

The left hand side expression of (42) becomes

$$
\begin{aligned}
& \nabla_{k}\left(V^{m}{ }_{i} V_{s}^{l}\right) C_{m l}+V^{m}{ }_{i} V^{l}{ }_{s}\left(\Gamma^{q}{ }_{k m} C_{q l}+\Gamma^{q}{ }_{k l} C_{m q}\right)= \\
& =\nabla_{k}\left(V^{m}{ }_{i} V_{s}^{l}\right) C_{m l}+V^{m}{ }_{i} V_{s}^{l}\left(\frac{\partial C_{m l}}{\partial x^{k}}\right)=2 \widetilde{Q}_{k s i} .
\end{aligned}
$$

The last equality in (50) is a direct consequence of the metric property of the connection $\nabla_{\Gamma}$, characterized by Formula (35).

The symmetry of the appropriate fields, written in (46) as well as in (47), follows as a consequence of (50).

Proposition 7. Formulas (43) and (44) together with (43) lead to the equality

$$
\begin{aligned}
& \nabla_{k} \mathbf{F}^{*} \circ \mathbf{F}+\mathbf{F}^{*} \circ \nabla_{k} \mathbf{F}=2\left\{\mathbf{C} \circ(\mathcal{A}-\mathbf{\Gamma})\left(\frac{\partial}{\partial x^{k}}\right)\right\}^{S}+2 \mathbf{Q}(k \cdot), \text { where } \\
& \mathbf{Q}(k \cdot)=Q_{k n j} d x^{n} \otimes d x^{j} \in \mathcal{T}^{0}{ }_{2}(\mathcal{B}), \quad \forall k \in\{1,2,3\} .
\end{aligned}
$$




\subsection{Decomposition Theorem of the Metric Connection}

The abstract result concerning the representation of the so-called connection coefficient, specific to a continuous deformable body with microstructure, can now be formulated.

Theorem 1. Let $\left(\mathcal{B}, \mathbf{C}, \nabla_{\Gamma}\right)$ be the set of the differential body manifold, $\mathcal{B}$, endowed with the metric $\mathrm{C}$, and a connection, $\nabla_{\Gamma}$, with the coefficient connection denoted by $\Gamma$.

Let $\left(\mathcal{B}_{t}, \mathbf{g}, \nabla_{\widetilde{\gamma}}\right)$ be the set of the differential body manifold at the moment $t, \mathcal{B}_{t}$, endowed with the metric $\mathbf{g}$ and the connection $\nabla_{\widetilde{\gamma}}$, with the coefficient connection denoted by $\widetilde{\gamma}$.

Under the hypotheses:

- The connection $\nabla_{\boldsymbol{\Gamma}}$ is metric;

- $\quad$ There exists an anholonomic $C^{2}$ diffeomorphism $\mathbf{F}(\cdot, t): \mathcal{T}(\mathcal{B}) \longrightarrow \mathcal{T}\left(\mathcal{B}_{t}\right)$, such that the metric tensor $\mathbf{C}(\cdot, t)=\mathbf{F}^{*}(\cdot, t) \circ \mathbf{F}(\cdot, t)$,

the following exist:

a. a connection $\mathcal{A}$ compatible with the anholonomic diffeomorphism $\mathbf{F}$, i.e., in the gradient notation $\mathcal{A}=\mathbf{F}^{-1} \nabla \mathbf{F}$.

b. $\quad \mathbf{Q} \in \mathcal{T}^{0}{ }_{3}(\mathcal{B})$, such that $\mathbf{Q u}$ is a second-order symmetric tensor $\forall \mathbf{u} \in \mathcal{T}(\mathcal{B})$;

c. $\mathbf{\Omega} \in \mathcal{T}_{3}^{0}(\mathcal{B})$ such that $\mathbf{\Omega} \mathbf{u} \in \mathbf{\Omega}^{2}(\mathcal{B})$, i.e., $\mathbf{\Omega} \mathbf{u}$, is 2-form $\forall \mathbf{u} \in \mathcal{T}(\mathcal{B})$, such that

$$
\Gamma=\mathcal{A}+\mathbf{C}^{-1}(\mathbf{Q}+\mathbf{\Omega})
$$

The tensor fields $\mathbf{Q}$ and $\mathbf{\Omega}$ are expressed as

$$
\begin{aligned}
& \mathbf{Q}=Q_{k n j} d x^{n} \otimes d x^{j} \otimes d x^{k}, \\
& \mathbf{\Omega}=\mathbf{\Omega}_{k i j} d x^{i} \Lambda d x^{j} \otimes d x^{k} .
\end{aligned}
$$

In the relation (52), the tensor type representation (32) associated with the connection coefficients is introduced.

Proof. By this hypothesis, the connection $\nabla_{\Gamma}$ is metric. Thus, the covariant derivative of the metric tensor is vanishing, namely $\nabla_{k} \mathbf{C}(\mathbf{p}, t)=0$ (see formula (35)). Taking into account the relationship (25), the covariant derivative of $\mathbf{C}(\mathbf{p}, t)$ allows the expression:

$$
\nabla_{k} \mathbf{C}(\mathbf{p}, t)=\nabla_{k} \mathbf{F}^{*} \circ \mathbf{F}+\mathbf{F}^{*} \circ \nabla_{k} \mathbf{F} \equiv 0 .
$$

From (51) together with the formula (54), the following representation is derived:

$$
2\left\{\mathbf{C} \circ(\mathcal{A}-\mathbf{\Gamma})\left(\frac{\partial}{\partial x^{k}}\right)+\mathbf{Q}(k \cdot)\right\}^{S} \equiv 0,
$$

due to the symmetry of $\mathbf{Q}(k \cdot)$.

From (54), we read that the second-order tensor field that is written inside the bracket is a skew-symmetric second-order field. Consequently, there exists a two-form on $\mathcal{B}$, i.e.,

$$
\boldsymbol{\Omega}_{k}=\boldsymbol{\Omega}_{k i j} d x^{i} \Lambda d x^{j},
$$

which characterizes the skew-symmetry mentioned above, namely

$$
\mathbf{C} \circ(\mathcal{A}-\mathbf{\Gamma})\left(\frac{\partial}{\partial x^{k}}\right)+\mathbf{Q}(k \cdot)=-\mathbf{\Omega}_{k}
$$

Remark 8. The result proved in Theorem 1 is in agreement with the statement: if $\Gamma$ and $\widetilde{\Gamma}$ are two connection coefficients on $\mathcal{B}$, then $\boldsymbol{\Gamma}-\widetilde{\boldsymbol{\Gamma}}$ is a tensor of type $(1,2)$, i.e., in $\mathcal{T}^{1}{ }_{2}(\mathcal{B})$. 
In order to emphasize the specificity of the representation for the connection coefficients given by (52), we recall the basic results (see, for instance, Schouten [35]).

Theorem 2. Let $\nabla_{\Gamma}$ be a metric connection, with connection coefficients $\Gamma$ and $\nabla_{\gamma}$ as the LeviCivita connection of the metric tensor $\mathbf{C}$. Both connections are defined on the differential manifold $\mathcal{B}$.

There exists a so-called contortion tensor $\mathbf{W} \in \mathcal{T}^{1}{ }_{2}(\mathcal{B})$, such that

$$
\Gamma=\gamma+\mathbf{W}
$$

1. The contortion $\mathbf{W}$ and the torsion $\mathbf{S}$ are tensors of the type $\mathcal{T}^{1}{ }_{2}(\mathcal{B})$, which determine each other by

$$
\begin{aligned}
& (\mathbf{W u}) \mathbf{v}=\frac{1}{2}(\mathbf{S u}) \mathbf{v}-\frac{1}{2} \mathbf{C}^{-1}\left[(\mathbf{C}(\mathbf{S v}))^{T} \mathbf{u}+(\mathbf{C}(\mathbf{S u}))^{T} \mathbf{v}\right], \quad \text { and } \\
& (\mathbf{S u}) \mathbf{v}=(\mathbf{W u}) \mathbf{v}-(\mathbf{W v}) \mathbf{u}, \quad \mathbf{u}, \mathbf{v} \in \mathcal{T}(\mathcal{B})
\end{aligned}
$$

taking into account the definition of the torsion $\mathbf{S}$,

$$
(\mathbf{S u}) \mathbf{v}=(\Gamma \mathbf{u}) \mathbf{v}-(\Gamma \mathbf{v}) \mathbf{u}, \forall \mathbf{u}, \mathbf{v} \in \mathcal{T}(\mathcal{B}) .
$$

2. The following skew-symmetries hold:

$$
(\mathbf{S u}) \mathbf{v}=-(\mathbf{S v}) \mathbf{u}, \quad(\overline{\mathbf{W}} \mathbf{u})^{T}=-(\overline{\mathbf{W}} \mathbf{u}), \quad \text { where } \quad \overline{\mathbf{W}}=\mathbf{C W} \in \mathcal{T}^{0}{ }_{3}(\mathcal{B}) .
$$

$\forall \mathbf{u}, \mathbf{v} \in \mathcal{T}(\mathcal{B})$.

To see the difference between the Schouten result and the decomposition derived in Theorem 1, we write Theorem 2, which is related to the decomposition of a metric connection when the metric is given. In Theorem 1, there exists the hypothesis that the metric tensor is associated with an anholonomic diffeomorphism.

\section{Plastic Connection for Material with Micro-Structure}

In the elasto-plastic description of the material body, we assume that

- $\quad \mathcal{B}$, and $\mathcal{B}_{t}$, are differentiable manifolds, at any time $\mathrm{t}$;

- $\quad$ the existence of a differentiable manifold (time dependent, but we omit the $t$ in the description for the sake of simplicity), $\widetilde{\mathcal{B}}$, called intermediate configuration, such that $\mathcal{B}$ and $\widetilde{\mathcal{B}}$ are $C^{2}-$ diffeomorphic; i.e., there exists a diffeomorphism, say $f: \mathcal{B} \longrightarrow \widetilde{\mathcal{B}}$, of the class $C^{2}$, for all $\mathbf{X} \in \mathcal{B}$, and at every time $t$;

- $\quad$ the deformation gradient $\mathbf{F}$ is induced by the motion function, i.e., $\mathbf{F}(\cdot, t)=d \chi(\cdot, t)$ : $\mathcal{T}_{\mathbf{X}}(\mathcal{B}) \longrightarrow \mathcal{T}_{\mathbf{x}}\left(\mathcal{B}_{t}\right), \quad \mathbf{x}=\chi(\mathbf{X}, t)$;

- $\quad$ the existence of the plastic and elastic $C^{1}$ - non-induced diffeomorphisms, $\mathbf{F}^{p}$ and $\mathbf{F}^{e}$, (called distortions),

$$
\begin{aligned}
& \mathbf{F}^{p}(\mathbf{X}, t): \mathcal{T}_{X}(\mathcal{B}) \longrightarrow \mathcal{T}_{\mathbf{q}}(\widetilde{\mathcal{B}}), \quad \text { linear, } \\
& \mathbf{F}^{e}(\mathbf{X}, t): \mathcal{T}_{\mathbf{q}}(\widetilde{\mathcal{B}}) \longrightarrow \mathcal{T}_{\mathbf{X}}\left(\mathcal{B}_{t}\right), \quad \text { linear } \quad \mathbf{F}^{e}=\mathbf{F} \circ\left(\mathbf{F}^{p}\right)^{-1} .
\end{aligned}
$$

Remark 9. Consequently, $\mathbf{F}^{p}$ and $\mathbf{F}^{e}$ are involved in the multiplicative decomposition of the deformation gradient

$$
\mathbf{F}=\mathbf{F}^{e} \circ \mathbf{F}^{p}
$$


The following representations for the plastic distortion, $\mathbf{F}^{p}$, and its adjoint, $\mathbf{F}^{p *}$, hold, namely

$$
\begin{aligned}
& \mathbf{F}^{p}(\mathbf{X}, t): \mathcal{T}_{X}(\mathcal{B}) \longrightarrow \mathcal{T}_{\mathbf{q}}(\widetilde{\mathcal{B}}), \quad \mathbf{F}^{p}(\mathbf{X}, t)=\left(\mathbf{F}^{p}\right)^{i}{ }_{j} \frac{\partial}{\partial y^{i}} \otimes d x^{j} \\
& \mathbf{F}^{p *}(\mathbf{X}, t): \mathcal{T}_{\mathbf{q}}^{*}(\widetilde{\mathcal{B}}) \longrightarrow \mathcal{T}_{\mathbf{X}}^{*}(\mathcal{B}), \quad\left(\mathbf{F}^{p}\right)^{*}(\mathbf{X}, t)=\left(\mathbf{F}^{p}\right)^{k}{ }_{s} d x^{\mathcal{S}} \otimes \frac{\partial}{\partial y^{k}}
\end{aligned}
$$

The plastic metric tensor on $\mathcal{B}$ is defined as

$$
\mathbf{C}^{p}=\mathbf{F}^{p *} \circ \mathbf{F}^{p}
$$

and $\mathbf{C}^{p}(\mathbf{X}, t)$ induces the metric $\mathbf{g}^{p}(\mathbf{q}, t)$ on $\mathcal{T}_{\mathbf{q}}(\widetilde{\mathcal{B}})$ by $\mathbf{F}^{p}$, which is given by

$$
\mathbf{g}^{p}(\mathbf{q}, t)=g_{i j}^{(p)(\mathbf{q}, t)} d y^{i} \otimes d y^{j}, \quad \mathbf{g}_{i j}^{(p)}=\left(\left(\mathbf{F}^{p}\right)^{-1}\right)^{s}{ }_{i} C_{s l}^{(p)}\left(\left(\mathbf{F}^{p}\right)^{1}\right)^{l}{ }_{j} .
$$

Here, $C_{i j}^{(p)}$ are the coordinate components of the metric tensor $\mathbf{C}^{p}$.

We apply the abstract result, Theorem 1, concerning the representation of the so-called connection coefficient, specific to an elasto-plastic continuous deformable body with microstructure.

Theorem 3. Let $\left(\mathcal{B}, \boldsymbol{C}^{p}, \nabla_{\Gamma^{p}}\right)$ be the set of the differential body manifold, $\mathcal{B}$, endowed with the metric $\mathbf{C}^{p}$, and a connection, $\nabla_{\Gamma^{p}}$, with the coefficient connection denoted by $\boldsymbol{\Gamma}^{p}$.

Let $\left(\widetilde{\mathcal{B}}, \mathbf{g}^{p}, \nabla_{\widetilde{\gamma}}\right)$ be the set of $\mathcal{B}_{t}$ - the differential manifold representing the intermediate configuration, at the moment $t$ endowed with the metric $\mathbf{g}$, and the connection, $\nabla_{\widetilde{\gamma}}$, with the coefficient connection denoted by $\widetilde{\gamma}$.

Under the hypotheses:

- The connection $\nabla_{\boldsymbol{\Gamma}^{p}}$ is $\mathbf{C}^{p}-$ metric;

- $\quad$ There exists a $C^{2}$ diffeomorphism $\mathbf{F}^{p}(\cdot, t): \mathcal{T}(\mathcal{B}) \longrightarrow \mathcal{T}(\widetilde{\mathcal{B}})$, such that the metric tensor is given by $\mathbf{C}^{p}(\cdot, t)=\mathbf{F}^{p *}(\cdot, t) \circ \mathbf{F}^{p}(\cdot, t)$;

- $\quad$ The metric $\mathbf{g}^{p}$ is induced from $\mathbf{C}^{p}$ by $\mathbf{F}^{p}$ via the Formula (66).

The following representation for the plastic connection coefficients is provided as

$$
\boldsymbol{\Gamma}^{p}=\mathcal{A}^{p}+\left(\mathbf{C}^{p}\right)^{-1}\left(\mathbf{Q}^{p}+\mathbf{\Omega}^{p}\right)
$$

Here,

a. $\mathcal{A}^{p}$ is a connection compatible with the diffeomorphism $\mathbf{F}^{p}$, i.e., in the gradient notation $\mathcal{A}^{p}=\left(\mathbf{F}^{p}\right)^{-1} \nabla \mathbf{F}^{p}$

b. $\quad \mathbf{Q}^{p} \in \mathcal{T}^{0}{ }_{3}(\mathcal{B})$, such that $\mathbf{Q}^{p} \widetilde{\mathbf{u}}$ is a second-order symmetric tensor $\forall \widetilde{\mathbf{u}} \in \mathcal{T}(\mathcal{B})$;

c. $\mathbf{\Omega}^{p} \in \mathcal{T}_{3}^{0}(\mathcal{B})$ such that $\mathbf{\Omega}^{p} \widetilde{\mathbf{u}} \in \mathbf{\Omega}^{2}(\mathcal{B})$, i.e., $\mathbf{\Omega}^{p} \widetilde{\mathbf{u}}$, is two-form $\forall \widetilde{\mathbf{u}} \in \mathcal{T}(\mathcal{B})$.

A similar decomposition to that given in (67) can be seen in Minagawa [30].

Despite working with the intermediate configuration $\widetilde{\mathcal{B}}$ viewed as a smooth differential manifold (motivated by the simplification in the notations), we have in mind a material neighbourhood of a given material point, $\mathcal{N}$, cut off from the deformed body configuration, and which is free of the loading, by a certain relaxation process. As we mentioned, it is not justified from a mathematical point of view to associate a differentiable manifold structure to the assembly of these locally relaxed configurations. Hence, when we refer to the assembly of locally relaxed configurations, the hypothesis of the vector bundle, whose basis is the differential manifold of the body in reference configuration, seems to be more adequate (from the differential geometry point of view) than the previous assumption. In a previous paper, we adopt a new definition for the plastic distortion to be a diffeomorphism acting from the tangent vectors on the differential manifold $\mathcal{B}$ on a vector bundle, $\mathcal{E}$. 


\section{Measure of Microstructural Defects}

We characterize the lattice defects in terms of the elements that enter the decomposition of the so-called plastic connection. The defects are treated as continuously and locally distributed in the body. Geometrical properties of the non-Riemannian connection, in their different approaches, are considered to characterize dislocations (Bilby [29] and Noll [20]), disclinations (De Wit [10,26]), and point defects (Minagawa [30] and Kröner [11].

\subsection{Density of Dislocations}

In contrast with the deformation gradient, $\mathbf{F}$, the plastic distortion $\mathbf{F}^{p}$ is not integrable. Only the non-integrability of $\mathbf{F}^{p}$ is associated with defects such as dislocations. The nonintegrability condition for $\mathbf{F}^{p}$ means that, on a certain simple connected set, such as a part of the body, the skew-symmetric part of the third-order field $\mathcal{A}^{p}$, denoted here as $S k w \mathcal{A}^{p}$, is not vanishing. Starting from the definition of $S k w \mathcal{A}$ given for any third-order tensor field,

$$
\left(S k w \mathcal{A}^{p} \mathbf{u}\right) \mathbf{v} \equiv\left(\mathcal{A}^{p} \mathbf{u}\right) \mathbf{v}-\left(\mathcal{A}^{p} \mathbf{v}\right) \mathbf{u}, \quad \forall \mathbf{u}, \mathbf{v} \in \mathcal{T}(\mathcal{B}),
$$

we found that

$$
S k w \mathcal{A}^{p}=\left(\left(\mathbf{F}^{(p)}\right)^{-1}\right)^{m}{ }_{s}\left(\frac{\partial}{\partial x^{k}}\left(\left(F^{(p)}\right)^{s}{ }_{j}\right)-\frac{\partial}{\partial x^{j}}\left(\left(F^{(p)}\right)^{s}{ }_{k}\right)\right) \frac{\partial}{\partial x^{m}} \otimes d x^{j} \otimes d x^{k} .
$$

Remark 10. The tensor field $S k w \mathcal{A}^{p}$ can be a measure of dislocations, following Bilby [29] and Noll [20].

The third-order tensor field $S k w_{\mathcal{A}} \mathcal{A}$ is related to the second-order tensor $\alpha=\left(\mathbf{F}^{p}\right)^{-1} \operatorname{curl}\left(\mathbf{F}^{p}\right)$ (which is defined in [34], the geometrically necessary dislocation, GND-tensor) by

$$
((S k w \stackrel{(p)}{\mathcal{A}}) \mathbf{u}) \mathbf{v}=\left(\mathbf{F}^{p}\right)^{-1} \operatorname{curl}^{p}(\mathbf{u} \times \mathbf{v}),
$$

which are written for all vectors $\mathbf{u}$ and $\mathbf{v}$. Here, we use the definition for $\operatorname{curlF}^{p}$. By definition, $\operatorname{curl} \mathbf{A}$ is the second-order tensor field, which allows the given representation

$$
(\operatorname{curl} \mathbf{A})(\mathbf{u} \times \mathbf{v}):=\left(\frac{\partial A_{i j}}{\partial x^{k}}-\frac{\partial A_{i k}}{\partial x^{j}}\right) u^{k} v^{j} \mathbf{e}^{i}
$$

in a local basis, associated with a curvilinear coordinate system $\left\{x^{j}\right\}$.

Concerning the plastic connection, we account for two cases.

In the first case, case I, in the expression of the plastic connection (see Formula (67)), the so-called symmetric part of the third-order tensor field, $\mathbf{Q}^{p}$ is vanishing, i.e., $\mathbf{Q}^{p}=0$.

In the second case, case II, the full representation for the plastic connection, derived in Formula (67), is considered.

\subsection{Density of Disclinations}

Case I. We look at the body $\mathcal{B}$ with the geometric structure $\left(\mathcal{B}, \nabla_{\Gamma^{p}}, \mathbf{C}^{p}\right)$, in which $\Gamma^{p}$, written in (67), is replaced by

$$
\boldsymbol{\Gamma}^{p}=\mathcal{A}^{p}+\left(\mathbf{C}^{p}\right)^{-1} \mathbf{\Omega}^{p},
$$

- $\quad$ with a metric $\mathbf{C}^{p}=\mathbf{F}^{p *} \circ \mathbf{F}^{p}$, but under the hypothesis that the induced metric $\mathbf{g}^{(p)}$ (see (66)), is such that

$$
\mathbf{g}_{m n}^{(p)}(\mathbf{q}, t)=\left(F^{-1}\right)^{i}{ }_{m} C_{i j}(\mathbf{p}, t)\left(F^{-1}\right)^{j}{ }_{n} \equiv \delta_{m n}
$$

- $\quad$ with the crystal connection $\nabla_{\mathcal{A}}^{p}$ with the coefficient connection described by (40). 
In reference to (73), Clayton [26] notices that this implies either $\widetilde{\mathcal{B}}$ is a Euclidean space, or the basis vectors in $\widetilde{\mathcal{B}}$ are not tangent to any coordinate system, i.e., they correspond to some external reference frame.

The torsion of the connection considered in (72) is given by

$$
\left(\mathbf{S}^{p} \mathbf{u}\right) \mathbf{v}=\left(\mathcal{A}^{p} \mathbf{u}\right) \mathbf{v}-\left(\mathcal{A}^{p} \mathbf{v}\right) \mathbf{u}+\left(\mathbf{C}^{p}\right)^{-1}\left(\left(\mathbf{\Omega}^{p} \mathbf{u}\right) \mathbf{v}-\left(\mathbf{\Omega}^{p} \mathbf{v}\right) \mathbf{u}\right),
$$

written for all vectors $\mathbf{u}, \mathbf{v} \in \mathcal{T}(\mathcal{B})$. The tensor field $\mathbf{\Omega}^{p}$, which enters (74), is expressed as in (53) 2 and has the property

$$
\boldsymbol{\Omega}^{p} \mathbf{u}=\mathbf{\Omega}_{k n j}^{(p)} u^{k} d x^{n} \Lambda d x^{j}, \text { i.e., } \mathbf{\Omega}_{k n j}^{(p)}=-\mathbf{\Omega}_{k j n^{\prime}}^{(p)} \forall j, n .
$$

The expression of the Cartan torsion, $\mathbf{S}^{p}$, written in (74), is given by

$$
\mathbf{S}^{p}=\operatorname{Skw}(\mathrm{p})+\left(\mathbf{C}^{p}\right)^{-1} \operatorname{Skw}\left(\mathbf{\Omega}^{p}\right) .
$$

Remark 11. The tensor field $S k w\left(\boldsymbol{\Omega}^{p}\right)$ can be a measure of disclinations.

Using the tensorial representation given by Cleja-Tigoiu in [17], the following representation for $\mathbf{\Omega}^{p}$ can be derived:

$$
\left.\left(\boldsymbol{\Omega}^{p} \mathbf{u}\right) \mathbf{v}=(\boldsymbol{\Lambda} \mathbf{u}) \times \mathbf{v} \equiv((\boldsymbol{\Lambda} \times \mathbf{I}) \mathbf{u})\right) \mathbf{v}, \quad \text { defined } \quad \forall \mathbf{u}, \mathbf{v},
$$

in terms of the second order field $\boldsymbol{\Lambda}, \mathbf{I}$ being the identity second-order tensor.

Thus, the Cartan torsion (76) is given by

$$
\mathbf{S}^{p}=S k w \mathcal{A}+\left(\mathbf{C}^{p}\right)^{-1} S k w(\boldsymbol{\Lambda} \times \mathbf{I}),
$$

and it can be expressed in terms of the second-order torsion, denoted by $\mathcal{N}^{p}$,

$$
\begin{aligned}
& \mathcal{N}^{p}=\left(\mathbf{F}^{p}\right)^{-1} \operatorname{curl} \mathbf{F}^{p}+\left(\mathbf{C}^{p}\right)^{-1}\left((\operatorname{tr} \boldsymbol{\Lambda}) \mathbf{I}-(\boldsymbol{\Lambda})^{T}\right), \\
& \text { where } \quad \mathcal{N}^{p}(\mathbf{u} \times \mathbf{v})=\left(\mathbf{S}^{p} \mathbf{u}\right) \mathbf{v}, \quad \forall \mathbf{u}, \mathbf{v} .
\end{aligned}
$$

The following result is proved in $[17,36]$.

\subsection{Lattice Defects and Their Interplay}

In case II, the plastic connection is given by (67), with $\mathbf{Q}^{(p)}$ written as in (53), and such that the second-order tensor, $\mathbf{Q}^{(p)} \mathbf{u}$, is symmetric, namely

$$
\mathbf{Q}^{p}=Q_{k n j}^{(p)} d x^{n} \otimes d x^{j} \otimes d x^{k}, \quad Q_{k n j}^{(p)}=Q_{k j n}^{(p)} .
$$

If we compute the Cartan torsion attached to the plastic connection (67), the contribution due to $\mathbf{Q}^{p}$ has to be considered. As $\mathbf{Q}^{p} \mathbf{u}$ is a second-order symmetric tensor $\forall \mathbf{u} \in \mathcal{T}(\mathcal{B})$, we evaluate the appropriate contribution as follows:

$$
\left(\mathbf{Q}^{(p)} \mathbf{u}\right) \mathbf{v}-\left(\mathbf{Q}^{(p)} \mathbf{v}\right) \mathbf{u}=\left(Q_{k n j}^{(p)} u^{k_{v} j}-Q_{k n j}^{(p)} u^{j} v^{k}\right) d x^{n}=Q_{[k j] n}^{(p)} u^{k} v^{j} d x^{n} .
$$

The skew-symmetry with respect to the indices $\mathrm{k}$ and $\mathrm{j}$ is mentioned through the notation $Q_{[k j] n}^{(p)}$. Finally, we obtain

$$
S k w \mathbf{Q}^{(p)}=d x^{n} \otimes Q_{k j n}^{(p)}\left(d x^{j} \Lambda d x^{k}\right) .
$$


Proposition 8. The Cartan torsion, denoted by $\overline{\mathbf{S}}$, associated with the plastic connection defined by (67), is expressed in terms of

- $\quad$ the dislocation density $S k w \mathcal{A}$, given by (68);

- $\quad$ the disclination density $\operatorname{Skw}\left(\boldsymbol{\Omega}^{p}\right)$;

- $\quad$ the defect of non-metricity type (such as point defects, extra-matter, vacancy)), $S k w \mathbf{Q}^{(p)}$, written in (82) as

$$
\overline{\mathbf{S}}=S k w \mathcal{A})+\left(\mathbf{C}^{p}\right)^{-1}\left(S k w\left(\mathbf{Q}^{(p)}\right)+S k w \mathbf{\Omega}^{p}\right)
$$

Several particular cases can be emphasized in reference to the field $\mathbf{Q}^{(p)}$, for instance:

(i) The point defect characterized by

$$
\begin{aligned}
& \mathbf{Q}^{(p)}=\hat{\mathbf{q}} \otimes \mathbf{C}, \quad \text { with } \hat{\mathbf{q}} \text { a 1-form, i.e., } \quad \hat{\mathbf{q}}=q_{n} d x^{n}, \\
& \quad \text { or in component representation as } \\
& \mathbf{Q}^{(p)}=q_{n} C_{k j} d x^{n} \otimes d x^{k} \otimes d x^{j} .
\end{aligned}
$$

In this case $S k w \mathbf{Q}^{(p)}=0$; thus, no contribution to the torsion follows.

(ii) The gradient-type defect occurs if there exists a symmetric, second-order field, $\mathbf{Q}$, such that

$$
\mathbf{Q}^{p}=\frac{\partial}{\partial x^{k}} \hat{Q}_{n j} d x^{n} \otimes d x^{j} \otimes d x^{k}, \quad \hat{Q}_{n j}=\hat{Q}_{j n} .
$$

In this case, by direct calculus, we obtain

$$
S k w \mathbf{Q}^{(p)}=\frac{\partial}{\partial x^{k}} \hat{Q}_{n j} d x^{n} \otimes\left(d x^{j} \Lambda d x^{k}\right) .
$$

Remark 12. The measures of the lattice defects are defined in Proposition 8 as third-order tensor fields. To justify the given definitions, we return to the classical framework of continuum mechanics. These densities can be rewritten in terms of second-order tensor fields, and thus comparison to the other definitions becomes possible, and their physical meanings can be emphasized at once.

Remark 13. If the crystal connection $\mathcal{A}^{p}$ is symmetric, i.e., $S k w \mathcal{A}^{p}=0$, then the so-called geometrically necessary dislocations are absent, i.e., GND-tensor $=0$.

The connection $\mathcal{A}^{p}$, called the crystal connection, following Le and Stumpf [31], is an integrable connection. Thus, the Riemannian curvature tensor is vanishing.

The connections associated with non-zero curvature are able to describe the presence of rotational defects in the lattice, defects that were interpreted as disclinations by De Wit [10].

Contrarily, if we compute the curvature tensor, $\mathcal{R}^{\Lambda}$, formed with the third-order tensor, $\mathbf{\Lambda} \times \mathbf{I}$, we proved in [17] that there exists a second-order tensor $\mathbf{r}^{\Lambda}$, such that

$$
\begin{aligned}
& \mathbf{r}^{\Lambda}=\operatorname{curl} \mathbf{\Lambda}+(\operatorname{Adj} \boldsymbol{\Lambda})^{T}, \\
& \left.\left(\mathcal{R}^{\Lambda} \mathbf{u}\right) \mathbf{v}\right) \mathbf{w} \cdot \mathbf{z}=\mathbf{r}^{\Lambda}(\mathbf{u} \times \mathbf{v}) \cdot(\mathbf{w} \times \mathbf{z}),
\end{aligned}
$$

for all vectors $\mathbf{u}, \mathbf{v}, \mathbf{w}, \mathbf{z}$. Here, $(\operatorname{Adj} \boldsymbol{\Lambda})^{T}(\mathbf{u} \times \mathbf{v})=\mathbf{\Lambda} \mathbf{u} \times \mathbf{\Lambda} \mathbf{v}$.

The Cartan torsion, $\overline{\mathbf{S}}$, defined by (83) is written under the form

$$
\overline{\mathbf{S}}=S k w \mathcal{A}+\left(\mathbf{C}^{p}\right)^{-1} S k w(\boldsymbol{\Lambda} \times \mathbf{I})+\left(\mathbf{C}^{p}\right)^{-1} S k w \mathbf{Q}^{(p)}
$$

We conclude that $S k w \mathcal{A}$ can be related to defects such as dislocations, while $S k w\left(\mathbf{\Omega}^{p}\right)$ can be interpreted to be responsible for defects such as disclination. Concerning the last 
term in the expression of Cartan torsion written in (88), let us introduce a second-order tensor, $\Omega^{Q}$, such that

$$
\left(\left(S k w \mathbf{Q}^{(p)}\right) \mathbf{u}\right) \mathbf{v}=\mathbf{\Omega}^{Q}(\mathbf{u} \times \mathbf{v})
$$

following the procedure proposed in [34]. Consequently, the second-order torsion tensor, $\overline{\mathcal{N}}$, associated with Cartan torsion, written in (88), via Formula (79) 1 , reads

$$
\begin{aligned}
& \overline{\mathcal{N}}=\left(\mathbf{F}^{p}\right)^{-1} \operatorname{curlF}^{p}+\left(\mathbf{C}^{p}\right)^{-1}\left((\operatorname{tr} \boldsymbol{\Lambda}) \mathbf{I}-(\boldsymbol{\Lambda})^{T}+\mathbf{\Omega}^{Q}\right) \\
& \text { where } \quad \overline{\mathcal{N}}^{p}(\mathbf{u} \times \mathbf{v})=(\overline{\mathbf{S}} \mathbf{u}) \mathbf{v}, \quad \forall \mathbf{u}, \mathbf{v} .
\end{aligned}
$$

Let us remark that, for the special case considered in (81),

$$
\Omega^{Q}=\frac{1}{2} \operatorname{curl} \hat{Q} .
$$

Remark 14. The physical characteristics can be attached to the defect variables, following Kröner, in the interpretation performed by de Wit [7] in linear approximation, as follows: $\hat{\mathbf{Q}}$ is introduced via Formula (85), and it is a symmetric second-order tensor. As it is related to a measure of nonmetricity, $\hat{\mathbf{Q}}$ is called the quasi-plastic strain (see also Kröner [32]). The second-order field $\mathbf{\Omega}^{Q}$ introduced via (89) can be called quasi-dislocation. To justify the attributed name, let us remark that the special case considered in (86) leads us to the Cartan torsion (see (90)), which contains $\operatorname{curl}^{p}$ and curl $\mathbf{Q}$ in a linear approximation. To paraphrase (for large deformation) De Wit [7], we concluded that each of these tensor fields "plays a valuable role in the understanding of the theory of extra-matter".

In order to take into account the interplay between defects, the following densities can be useful:

$$
\begin{aligned}
& \mathbf{D}_{1}=\operatorname{Skw} \underset{\mathcal{A}}{\mathcal{A}}+\left(\mathbf{C}^{p}\right)^{-1} \operatorname{Skw}(\mathbf{\Lambda} \times \mathbf{I}) \\
& \mathbf{D}_{2}=\operatorname{Skw} \underset{\mathcal{A}}{\mathcal{A}}+\left(\mathbf{C}^{p}\right)^{-1} \operatorname{Skw} \mathbf{Q}^{(p)} \\
& \left.\mathbf{D}_{3}=\operatorname{Skw} \mathcal{A}\right)\left(\mathbf{C}^{p}\right)^{-1}\left(\operatorname{Skw} \mathbf{Q}^{(p)}+\operatorname{Skw}(\mathbf{\Lambda} \times \mathbf{I})\right) .
\end{aligned}
$$

The interplay between dislocations and disclinations is measured by $\mathbf{D}_{1}$, while the interplay between dislocations and defect variable $\mathbf{Q}^{(p)}$ is described by $\mathbf{D}_{2}$. Finally, the possible interplay between dislocations, disclinations, and extra-matter defects can be characterized by $\mathbf{D}_{3}$.

\section{Conclusions}

Based on the theorem concerning the decomposition of the plastic connection, measures of lattice defect densities are introduced as third-order tensor fields. The definition of plastic distortion as an incompatible diffeomorphism is based on the existence of the intermediate configuration considered to be a differential manifold. The metric induced by the plastic distortion on the intermediate configuration is a key point in the theory. No assumptions concerning the non-metricity of the plastic connection is done. As a direct consequence of the metric induced by the plastic distortion, defects such as extra-mater are infered in model. If the metric on the intermediate configuration is Euclidean, then the non-metric property of the connection does not result. All types of lattice defects, dislocations, disclinations, and point defects can be described in terms of the densities related to the elements that characterize the decomposition theorem for plastic connection. As a novelty, the measure of the interplay of the possible lattice defects is introduced via the Cartan torsion tensor. We justify the given definitions by comparing the proposed measure of defects with their counterparts corresponding to classical framework of continuum mechanics. Thus, their physical meanings can be emphasized at once. 
Funding: This research received no external funding.

Conflicts of Interest: The author declares no conflict of interest.

\section{References}

1. Mandel, J. Plasticité Classique et Viscoplasticité; CISM-Udine; Springer: Vienna, Austria; New York, NY, USA, 1972.

2. Teodosiu, C. A dynamic theory of dislocations and its applications to the theory of the elastic- plastic continuum. In Fundamental Aspects of Dislocation Theory; Simmons, J.A., De Witt, R., Bullough, R., Eds; National Bureau of Standards (U.S.): Gaithersburg, MD, USA, 1970; Volume 317, pp. 837-876.

3. Cleja-Ţigoiu, S.; Soós, E. Elastoviscoplastic models with relaxed configurations and internal state variables. Appl. Mech. Rev. 1990, 43, 131-151. [CrossRef]

4. Romanov, A.E. Mechanics and physics of disclinations in solids. Europ. J. Mech. A/Solids 2003, 22, 727-741. [CrossRef]

5. Cleja-Ţigoiu, S.; Paşcan, R.; Ţigoiu, V. Interplay between continuous dislocations and disclinations in elasto-plasticity. Int. J. Plast. 2016, 68, 88-110. [CrossRef]

6. Cleja-Ţigoiu, S.; Paşcan, R.; Tigoiu, V. Disclination based model of grain boundary in crystalline materials with microstructural defects. Int. J. Plast. 2019, 114, 227-251. [CrossRef]

7. De Wit, R. Theory of Disclinations: II. Continuous and Discrete Disclinations in Anisotropic Elasticity. J. Res. Nat. Bur. Stand. A Phys. Chem. 1973, 77A, 49-100. [CrossRef] [PubMed]

8. Fressengeas, C.; Taupin, V.; Capolungo, L. An elasto-plastic theory of dislocation and disclination field. Int. J. Solids Struct. 2011, 48, 3499-3509. [CrossRef]

9. Fressengeas, C.; Taupin, V.; Capolungo, L. Continuous modeling of structure of symmetric tilt boundaries. Int. J. Solids Struct. 2014, 51, 1434-1441. [CrossRef]

10. De Wit, R. A view of the relation between the continuum theory of lattice defects and non-Euclidean geometry in the linear approximation. Int. J. Eng. Sci. 1981, 19, 1475-1506. [CrossRef]

11. Kröner, E. The Differential geometry of Elementary Point and Line Defects in Bravais Crystals. Int. J. Theor. Phys. 1990, 29, 1219-1237. [CrossRef]

12. Cleja-Tigoiu, S. Anisotropic Damage in Elasto-plastic Materials with Structural Defects. In Multiscale Modelling in Sheet Metal Forming; Banabic, D., Ed.; Spinger International Publishing AG: Cham, Switzerland, 2016; pp. 301-351.

13. Cleja-Ţigoiu, S.; Ţigoiu, V. Continuous Model of Structural Defects in Finite Elasto-Plasticity; Éditions Universitaires Européenes: Paris, France, 2017; pp. 5-73.

14. Cleja-Tiigoiu, S. Finite Elasto-Plastic Models for Lattice Defects in Crystalline Materials. In Mathematical Modelling in Solid Mechanics, Advanced Structured Materials 69; dell'Isola, F., Sofonea, M., Steigmann, D., Eds; Springer Nature Singapore Pte Ltd.: Singapore, 2017; pp. 43-58.

15. Cleja-Tुigoiu, S. Evolution Equation for Defects in Finite Elasto-Plasticity. In Generalized Models and Non-classical Approaches in Complex Materials 1, Advanced Structured Materials 89; Altenbach, H., Pouget, J., Rousseau, M., Collet, B., Michelitsch, T., Eds.; Springer International Publishing AG.: Cham, Switzerland, 2018; pp. 179-201.

16. Gurtin, M.E.; Fried, E.; Anand, L. The Mechanics and Thermodynamics of Continua; Cambridge University Press: Cambridge, MA, USA, 2010.

17. Cleja-Ţigoiu, S. Elasto-plastic materials with lattice defects modelled by second order deformations with non-zero curvature. Int. J. Fract. 2010, 166, 61-75. [CrossRef]

18. Nakahara, N. Geometry, Topology and Physics, 2nd ed.; Taylor \& Franci: New York, NY, USA; London, UK, 2003.

19. Lee, J.M. Introduction to Smooth Manifolds; Springer Science+Business Media: New York, NY, USA, 2013.

20. Noll, W. Materially Uniform Simple Bodies with Inhomogeneities. Arch. Rat. Mech. Anal. 1967, 27, 1-32. [CrossRef]

21. Wang, C.C. On the Geometric Structure of Simple Bodies, a Mathematical Foundation for the Theory of Continuous Distributions of Dislocations. Arch. Rat. Mech. Anal. 1967, 27, 33-94. [CrossRef]

22. Steinmann, P. Geometrical Foundations of Continuum Mechanics, An application to First-and Second-Order Elasticity and Elasto-Plasticity; Springer: Berlin/Heidelberg, Gremany, 2015.

23. Epstein, M. The Geometry Language of Continuum Mechanics; Cambridge University Press: Cambridge, MA, USA, 2010.

24. Clayton, J.D. Defects in Nonlinear Elastic Crystals: Differential Geometry, Finite Kinematics, and Second-Order Analytical Solutions. ZAMM-J. Appl. Math. Mech./Z. Angew. Math. Und Mech. 2015, 95, 476-510. [CrossRef]

25. Yavari, A.; Goriely, A. Riemann-Cartan Geometry of Nonlinear Disclination Mechanics. Math. Mech. Solids 2012, 18, 91-102. [CrossRef]

26. Clayton, J.D. Nonlinear Mechanics of Crystals; Solid Mechanics and Its Applications 177; Springer: Berlin/Heidelberg, Germany, 2011.

27. Yavari, A.; Goriely, A. Riemann-Cartan Geometry of Nonlinear Dislocation Mechanics. Arch. Rat. Mech. Anal. 2012, 205, 59-118. [CrossRef]

28. Cartan, H. Calcul Différentiel. Formes Différentielles; Hermann: Paris, France, 1967.

29. Bilby, B.A. Continuous distribution of dislocations. In Progress in Solid Mechanics; Sneddon, I.N., Hill, R., Eds.; North-Holland Publishing Company: Amsterdam, The Netherlands, 1960; pp. 329-398.

30. Minagawa, S. A non-Riemannian geometrical theory of imperfections in a Cosserat continuum. Arch. Mech. 1979, 31, 783-792. 
31. Le, E.; Stumpf, H. On the determination of crystal reference in nonlinear continuum theory of dislocations. Proc. R. Soc. Lond. A 1996, 452, 359-371.

32. Kröner, E. The internal mechanical state of solids with defects. Int. J. Solids Struct. 1992, 29, 1849-1857. [CrossRef]

33. Kleman, M.; Fridel, J. Disclinations, dislocations, and continuous defects: A reappraisal. Rev. Mod. Phys. 2008, 80, 61-115. [CrossRef]

34. Cleja-Ţigoiu, S. Disclinations and GND tensor effects on the multislip flow rule in crystal plasticity. Math. Mech. Solids 2020, 25, 1643-1676. [CrossRef]

35. Schouten, J. Ricci Calculus; Springer: Berlin, Germany, 1954.

36. Cleja-Ţigoiu, S. Material Forces in Finite Elastoplasticity with Continuously Distributed Dislocations. Int. J. Fract. 2007, 147, 67-81. [CrossRef] 